\title{
Preoperative serum docosahexaenoic acid level predicts prognosis of renal cell carcinoma
}

\author{
SHINSUKE TASAKI ${ }^{1,2}$, AKIO HORIGUCHI ${ }^{1}$, TAKAKO ASANO ${ }^{1}$, KENJI KURODA ${ }^{1}$, AKINORI SATO ${ }^{1}$, \\ JUNICHI ASAKUMA ${ }^{1}$, KEIICHI ITO ${ }^{1}$, TOMOHIKO ASANO ${ }^{1}$ and HIROTAKA ASAKURA ${ }^{2}$ \\ ${ }^{1}$ Department of Urology, National Defense Medical College, Tokorozawa, Saitama 359-8513; \\ ${ }^{2}$ Department of Urology, Saitama Medical University, Iruma, Saitama 350-0495, Japan
}

Received October 23, 2015; Accepted April 15, 2016

DOI: $10.3892 / \mathrm{mco} .2016 .890$

\begin{abstract}
The recent discovery and clinical development of targeted agents have expanded treatment options in metastatic renal cell carcinoma (RCC). However, metastatic RCC remains a lethal disease. Complete response is rare and treatment with targeted agents eventually fails in the majority of the patients. Therefore, there is a need for developing a prognostic tool and a novel therapeutic agent for RCC to improve the follow-up strategy after surgical treatment. Clinical data, including patient characteristics, serum fatty acid profile, clinicopathological parameters and clinical outcome, were obtained from 112 patients with RCC prior to surgical treatment. Preoperative fatty acid levels were grouped according to patient characteristics, such as performance status, body mass index or pathological parameters, and were analyzed using the Mann-Whitney U test. Cancer-specific survival in the high and low docosahexaenoic acid (DHA) level groups were compared using the Kaplan-Meier method. Cox proportional hazards models were applied to determine the independent prognostic factors associated with shortened cancer-specific survival. The serum DHA level in patients with metastasis was significantly lower compared with that in patients without metastasis $(\mathrm{P}=0.047)$. Low serum DHA level, presence of metastasis and cachexia were independent predictors of shortened cancer-specific survival in a multivariate Cox proportional hazard model $(\mathrm{P}=0.033$, hazard ratio $=4.43$ ). Patients with a serum DHA level below the median value exhibited significantly shorter cancer-specific survival compared with those with a higher serum DHA level ( $\mathrm{P}=0.008)$. Thus, according to our results, the preoperative serum DHA level may be able to predict the surgical
\end{abstract}

Correspondence to: Dr Shinsuke Tasaki, Department of Urology, National Defense Medical College, 3-2 Namiki, Tokorozawa, Saitama 359-8513, Japan

E-mail: shinsuketasaki@yahoo.co.jp

Key words: docosahexaenoic acid, renal cell carcinoma, metastasis, prognosis, prognostic biomarker outcome of RCC. However, this finding requires validation by large-scale prospective studies.

\section{Introduction}

Renal cell carcinoma ( $\mathrm{RCC}$ ) represents $\sim 3 \%$ of all adult cancers (1). Approximately $30 \%$ of RCC patients have metastatic disease at the time of diagnosis, and another 20-30\% develop metastases after surgical treatment (2). To date, no specific serum biomarker has been shown to improve the predictive accuracy of the current prognostic system for RCC; thus, preoperative prediction of disease progression after surgery is difficult (3). Developing a specific molecular biomarker to predict surgical outcome is crucial for elucidating the mechanism underlying metastasis and recurrence in RCC, resulting in discovery of novel therapeutic agents. The discovery and clinical development of targeted agents have expanded treatment options in metastatic RCC. However, metastatic RCC remains a lethal disease. Complete response is rare and treatment with targeted agents eventually fails in the majority of the patients $(2,3)$. Therefore, there is an urgent need for developing a prognostic tool and a novel therapeutic agent for RCC.

The omega-3 fatty acids (FAs), including $\alpha$-linolenic acid (ALA), eicosapentaenoic acid (EPA) and docosahexaenoic acid (DHA), are long-chain polyunsaturated FAs with the first double bond on the third carbon from the methyl end of the chain. Omega-3 FAs are essential nutrients that cannot be synthesized in the body and must be obtained from the diet. EPA and DHA are abundant in fish oil (4) and fish oil supplements and fatty fish, such as salmon and tuna, are particularly rich sources of FAs, which are important dietary components for health maintenance and disease prevention. Increased consumption of omega-3 FAs has been shown to be associated with a lower incidence of various types of cancer, including colon (5), breast $(6,7)$ and prostate cancer $(6,7)$. Omega-3 FAs have been shown to exert a variety of antitumor effects, such as antitumor angiogenesis (8), induction of cancer cell apoptosis (9), inhibition of tumor invasion and metastasis $(10,11)$, and attenuation of signaling pathways (12). The antitumor effects of omega-3 FAs have been suggested by clinical reports demonstrating that higher intakes of EPA and DHA reduced the risk of mortality in women treated for breast cancer $(13,14)$. 
The administration of DHA during anthracyclin-based chemotherapy against metastatic breast cancer improved the outcome of chemotherapy, suggesting that omega-3 FAs may be effective adjuvants (15).

In the present study, we investiagted the serum DHA level in RCC patients and evaluated the effect of DHA level on pathological parameters and surgical outcome.

\section{Patients and methods}

Patients. A total of 112 patients who underwent surgical treatment for RCC at the National Defense Medical College (Tokorozawa, Japan) between July, 2003 and April, 2009 were included in this study. The characteristics of the patients examined are summarized in Table I. Cachexia is defined as a condition with weight loss and/or hypoalbuminemia $(\leq 3.8 \mathrm{mg} / \mathrm{dl})$ and/or anorexia and malaise prior to surgical treatment. Clinical staging was performed according to the 2009 TMN staging system, using computed tomography (CT) scans of the chest, abdomen and pelvis. All tumor tissues were evaluated in terms of pathological staging and histological grading according to the TNM and the 2004 WHO classification of the renal tumors of the adults (16). Disease progression was defined as evidence of recurrence or metastasis on periodic CT of the chest, abdomen and pelvis, or physical examinations. This study was conducted following permission of the Ethics Committee of our institute.

Serum FA profile. The levels and distribution profile of FAs in serum samples collected in the morning of the day of operation were measured by a transesterification method described previously (17). In brief, FA methyl esters were chromatographed on a 30x0.25 mm internal diameter DB-23 column (J\&W Scientific, Santa Clara, CA, USA), with a film thickness of $0.25 \mathrm{~mm}$ and a cyanopropyl/polysiloxane phase. Analysis was performed on a Dani 3800 GC programmed temperature vaporizer system (the injector temperature was raised from 50 to $250^{\circ} \mathrm{C}$ within 9-50 sec) equipped with a flame ionization detector (detector temperature, $260^{\circ} \mathrm{C}$ ) and a Shimadzu C-R1B integration unit (all from Shimadzu, Kyoto, Japan). The carrier gas was helium, flowing at $3 \mathrm{ml} / \mathrm{min}$. The injected sample volume was $1 \mathrm{ml}$ and the split rate was 1:10 (solvent split). The column temperature was increased from 130 to $240^{\circ} \mathrm{C}$ at $2^{\circ} \mathrm{C} / \mathrm{min}$. The peak quantification was based on peak area comparison with the internal standard (17:0,100 mg). The concentration of DHA as well as the proportion of DHA as a percentage of the total FA content were indicated. We examined the proportion as well as the concentration of the serum DHA in preoperative patients, as both have been shown to be significant predictors in patients with breast cancer or neuroblastoma $(15,18)$.

Statistical analysis. The results are expressed as mean value \pm standard error for three independent experiments. The Mann-Whitney U test was used to compare the serum levels of the FA profile in different groups. Cancer-specific survival was evaluated by the Kaplan-Meier method, and survival differences were compared using the log-rank test. Univariate and multivariate Cox proportional hazards models were used to assess the effect of independent predictors on time-to-event outcomes. P-values $<0.05$ were considered to indicate statistically significant differences.
Table I. Patient characteristics prior to surgical treatment.

\begin{tabular}{|c|c|c|}
\hline Characteristics & No. & $\%$ \\
\hline \multicolumn{3}{|l|}{ Age, years } \\
\hline Mean (median) & $61.2(63)$ & \\
\hline Range & $36-82$ & \\
\hline \multicolumn{3}{|l|}{ Gender } \\
\hline Male & 88 & 78.57 \\
\hline Female & 24 & 21.43 \\
\hline \multicolumn{3}{|l|}{ pT stage } \\
\hline T1a & 50 & 44.64 \\
\hline T1b & 31 & 27.68 \\
\hline $\mathrm{T} 2 \mathrm{a}$ & 10 & 8.93 \\
\hline $\mathrm{T} 2 \mathrm{~b}$ & 2 & 1.79 \\
\hline Т3a & 6 & 5.36 \\
\hline T3b & 12 & 10.71 \\
\hline $\mathrm{T} 3 \mathrm{c}$ & 0 & 0.00 \\
\hline $\mathrm{T} 4$ & 1 & 0.89 \\
\hline \multicolumn{3}{|l|}{$\mathrm{N}$ stage } \\
\hline N0 & 111 & 99.11 \\
\hline N1 & 1 & 0.89 \\
\hline \multicolumn{3}{|l|}{ M stage } \\
\hline M0 & 97 & 86.61 \\
\hline M1 & 15 & 13.39 \\
\hline \multicolumn{3}{|c|}{ Histological subtype } \\
\hline Clear cell & 105 & 93.75 \\
\hline Chromophobe & 4 & 3.57 \\
\hline Papillary & 3 & 2.68 \\
\hline \multicolumn{3}{|l|}{ Treatment } \\
\hline Radical & 97 & 86.61 \\
\hline Partial & 15 & 13.39 \\
\hline
\end{tabular}

\section{Results}

Preoperative FA levels and clinicopathological characteristics. The patient characteristics are summarised in Table I. Of the 112 patients, 81 presented with locally confined disease (T1-2), while 31 presented with locally advanced disease (T3-4). Of the 112 patients, 97 had no metastasis (M0), whereas 15 presented with metastasis (M1). Radical nephrectomy was performed in 97 cases and partial nephrectomy was performed in 15 cases. We first analyzed the associations between clinicopathological parameters and the serum level of omega-3 FAs in serum FA composition (Table II). The mean level of ALA in patients with T1-2 disease was significantly higher compared with that in patients with T3-4 disease $(\mathrm{P}=0.02)$. The mean level of DHA in patients without any metastasis (NOM0) was significantly higher compared with that in patients with metastatic disease $\left(\mathrm{N}^{+}\right.$and/or $\left.\mathrm{M}^{+}, \mathrm{P}=0.047\right)$.

Preoperative serum DHA levels and clinical outcome. Cancer-specific survival for patients stratified by preoperative serum DHA level is shown in Fig. 1. The 112 patients were divided into two groups depending on whether 
Table II. Association between serum omega-3 FA levels and pathological parameters.

\begin{tabular}{|c|c|c|c|c|c|c|c|c|c|}
\hline Parameters & No. & n-3 PUFA (\%) & P-value & $\operatorname{ALA}(\%)$ & P-value & $\operatorname{EPA}(\%)$ & P-value & DHA $(\%)$ & P-value \\
\hline Gender & & & 0.093 & & 0.248 & & 0.046 & & 0.274 \\
\hline Male & 88 & $6.84 \pm 0.22$ & & $0.71 \pm 0.02$ & & $2.17 \pm 0.10$ & & $3.95 \pm 0.14$ & \\
\hline Female & 24 & $6.02 \pm 0.43$ & & $0.66 \pm 0.04$ & & $1.74 \pm 0.19$ & & $3.62 \pm 0.26$ & \\
\hline ECOG PS & & & 0.258 & & 0.323 & & 0.335 & & 0.324 \\
\hline $0-1$ & 106 & $6.71 \pm 0.21$ & & $0.71 \pm 0.02$ & & $2.10 \pm 0.09$ & & $3.91 \pm 0.13$ & \\
\hline$>2$ & 6 & $5.70 \pm 0.86$ & & $0.62 \pm 0.08$ & & $1.71 \pm 0.39$ & & $3.37 \pm 0.53$ & \\
\hline $\mathrm{BMI}, \mathrm{kg} / \mathrm{m}^{2}$ & & & 0.639 & & 0.285 & & 0.288 & & 0.886 \\
\hline$<23$ & 59 & $6.75 \pm 0.28$ & & $0.68 \pm 0.03$ & & $2.17 \pm 0.13$ & & $3.89 \pm 0.17$ & \\
\hline$>23$ & 53 & $6.56 \pm 0.29$ & & $0.72 \pm 0.03$ & & $1.98 \pm 0.13$ & & $3.86 \pm 0.18$ & \\
\hline Cachexia & & & 0.345 & & 0.159 & & 0.649 & & 0.325 \\
\hline Absent & 103 & $6.71 \pm 0.21$ & & $0.71 \pm 0.02$ & & $2.09 \pm 0.09$ & & $3.91 \pm 0.13$ & \\
\hline Present & 9 & $6.02 \pm 0.71$ & & $0.61 \pm 0.07$ & & $1.94 \pm 0.32$ & & $3.47 \pm 0.43$ & \\
\hline T stage & & & 0.383 & & 0.02 & & 0.783 & & 0.383 \\
\hline $\mathrm{T} 1-2$ & 93 & $6.74 \pm 0.22$ & & $0.72 \pm 0.02$ & & $2.09 \pm 0.10$ & & $3.93 \pm 0.13$ & \\
\hline T3-4 & 19 & $6.27 \pm 0.49$ & & $0.61 \pm 0.04$ & & $2.02 \pm 0.22$ & & $3.64 \pm 0.29$ & \\
\hline Tumor necrosis & & & 0.121 & & 0.087 & & 0.246 & & 0.157 \\
\hline Negative & 74 & $6.88 \pm 0.25$ & & $0.72 \pm 0.02$ & & $2.16 \pm 0.11$ & & $4.00 \pm 0.15$ & \\
\hline Positive & 38 & $6.22 \pm 0.34$ & & $0.66 \pm 0.03$ & & $1.93 \pm 0.16$ & & $3.64 \pm 0.21$ & \\
\hline Metastasis & & & 0.074 & & 0.798 & & 0.222 & & 0.047 \\
\hline NOM0 & 97 & $6.80 \pm 0.21$ & & $0.70 \pm 0.02$ & & $2.13 \pm 0.09$ & & $3.97 \pm 0.13$ & \\
\hline $\mathrm{N}^{+}$and/or $\mathrm{M}^{+}$ & 15 & $5.75 \pm 0.54$ & & $0.69 \pm 0.05$ & & $1.79 \pm 0.25$ & & $3.26 \pm 0.33$ & \\
\hline Grade & & & 0.374 & & 0.537 & & 0.494 & & 0.392 \\
\hline G1-2 & 72 & $6.79 \pm 0.25$ & & $0.71 \pm 0.02$ & & $2.13 \pm 0.11$ & & $3.95 \pm 0.15$ & \\
\hline G3 & 40 & $6.42 \pm 0.34$ & & $0.69 \pm 0.03$ & & $1.99 \pm 0.15$ & & $3.74 \pm 0.20$ & \\
\hline Venous invasion & & & 0.164 & & 0.126 & & 0.372 & & 0.167 \\
\hline Negative & 65 & $6.89 \pm 0.26$ & & $0.73 \pm 0.02$ & & $2.15 \pm 0.12$ & & $4.02 \pm 0.16$ & \\
\hline Positive & 47 & $6.33 \pm 0.31$ & & $0.67 \pm 0.03$ & & $1.99 \pm 0.14$ & & $3.68 \pm 0.19$ & \\
\hline Histology & & & 0.419 & & 0.924 & & 0.691 & & 0.309 \\
\hline Clear cell & 105 & $6.62 \pm 0.21$ & & $0.70 \pm 0.02$ & & $2.07 \pm 0.09$ & & $3.84 \pm 0.13$ & \\
\hline Others & 7 & $7.29 \pm 0.80$ & & $0.71 \pm 0.08$ & & $2.22 \pm 0.36$ & & $4.36 \pm 0.49$ & \\
\hline
\end{tabular}

Values are presented as mean \pm standard error. PUFA, polyunsaturated fatty acids; ALA, $\alpha$-linolenic acid; EPA, eicosapentaenoic acid; DHA, docosahexaenoic acid; ECOG PS, Eastern Cooperative Oncology Group performance status; BMI, body mass index.

their DHA levels were above or below the median value and a survival curve for each group was generated. The cancer-specific survival of patients with a serum DHA level below the median value was significantly shorter compared with that in patients with a DHA level above the median value $(\mathrm{P}=0.008$; Fig. $1 \mathrm{~A})$. The effect of DHA level on the progression-free survival of the 97 patients without metastasis was evaluated by dividing the patients into two groups depending on whether their DHA levels were above or below the median value and a survival curve for each group was generated. The progression-free survival of patients with a serum DHA level below the median value was shorter compared with that in patients with a serum DHA level above the median value; however, the difference was not significant $(\mathrm{P}=0.144$; Fig. $1 \mathrm{~B})$. Other factors shown by univariate analysis to be predictive of shortened cancer-specific survival were cachexia $(\mathrm{P}<0.0001)$, more advanced tumor stage (pT3-4; $\mathrm{P}=0.0003)$, presence of tumor necrosis $(\mathrm{P}=0.002)$, higher tumor grade (grade $3 ; \mathrm{P}=0.0032)$, presence of metastasis $\left(\mathrm{N}^{+}\right.$and/or $\mathrm{M}^{+} ; \mathrm{P}<0.0001$, Table III) and low DHA level $(\mathrm{P}=0.0051)$. However, the multivariate Cox proportional hazards analysis of these significant prognostic factors revealed that cachexia $(\mathrm{P}=0.014)$, metastasis $(\mathrm{P}=0.023)$ and low DHA level $(\mathrm{P}=0.033)$ were independent predictors of shortened cancer-specific survival (Table III).

\section{Discussion}

Using the Cox proportional hazards model, we demonstrated that the preoperative low DHA level was one of the independent predictors of shortened cancer-specific survival in RCC patients who underwent surgical treatment. Furthermore, the DHA level in patients with metastatic disease was found to be significantly lower compared with that in patients without 

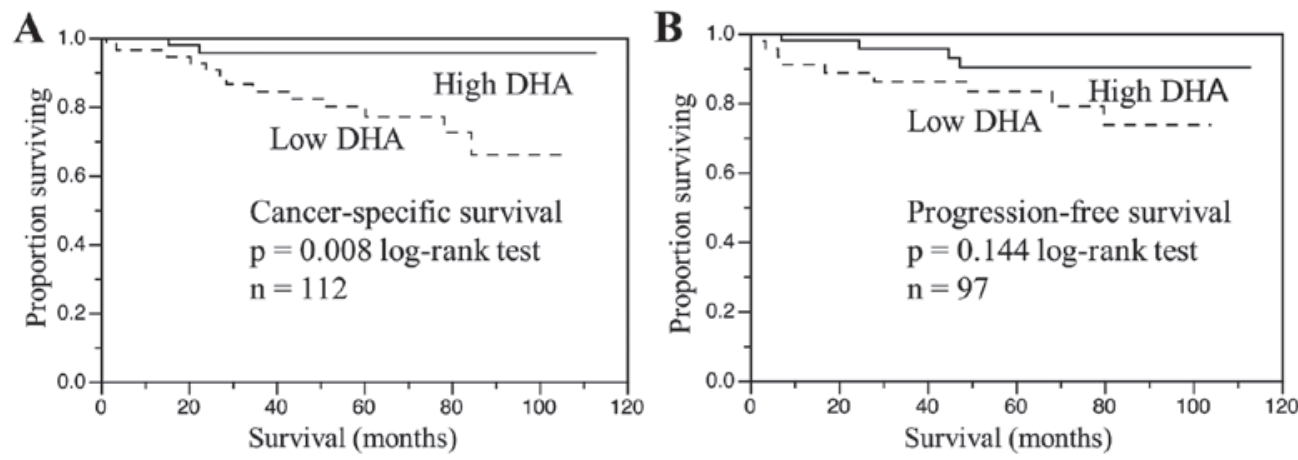

Figure 1. (A) Cancer-specific survival according to serum DHA level in patients with renal cell carcinoma. (B) Progression-free survival according to serum DHA levels in patients without metastatic disease. DHA, docosahexaenoic acid.

Table III. Risk factors for cancer-specific survival by univariate and multivariate analyses of Cox proportional hazards model.

\begin{tabular}{lccccc}
\hline & \multicolumn{2}{c}{ Univariate analysis } & & \multicolumn{3}{c}{ Multivariate analysis } \\
Factors & P-value & & P-value & HR & 95\% CI \\
\hline Cachexia (positive) & $<0.0001$ & & 0.014 & 6.29 & $1.48-28.37$ \\
T stage (T3-4) & 0.0003 & & 0.21 & 2.52 & $0.58-10.35$ \\
Tumor necrosis (positive) & 0.002 & & 0.644 & 1.46 & $0.29-7.45$ \\
Grade (G3) & 0.0032 & & 0.981 & 1.02 & $0.16-6.39$ \\
Metastasis ( $\mathrm{N}^{+}$and/or $\mathrm{M}^{+}$) & $<0.0001$ & & 0.023 & 4.71 & $1.23-23.76$ \\
DHA level (lower than median) & 0.0051 & & 0.033 & 4.43 & $1.11-30.25$ \\
\hline
\end{tabular}

HR, hazard ratio; CI, confidence interval; DHA, docosahexaenoic acid.

metastatic disease. To the best of our knowledge, this is the first report showing a association between serum DHA levels and pathological parameters and clinical outcome following surgical treatment in patients with RCC.

Although it has not been elucidated whether the low preoperative DHA level is a reason or a consequence of metastatic disease, resulting in poor cancer-specific survival, there is accumulating evidence indicating that DHA inhibits carcinogenesis (4), inflammation (19), angiogenesis (8) and metastasis (10) in animal and in vitro studies. It has been reported that serum levels of omega-3 FAs in patients with pancreatic cancer, lung cancer, or NHL are lower compared with those in healthy controls $(20,21)$. Moreover, pancreatic cancer or NHL patients with more advanced clinical stages have lower levels of serum omega-3 FAs $(21,22)$. In our study, there was a significant association between the presence of metastasis and decreased serum DHA level. DHA has been shown to reduce metastasis in animal experimental models $(10,11)$. DHA has been shown to prevent the migration and invasion of human MDA-MB-231 mammary cancer cells in vitro, and a DHA-rich fish oil diet prevents bone metastasis from breast cancer by reducing the expression of CD44, which has been identified as a molecular signature of cancer stem cells (10). A recent study has demonstrated that DHA inhibited vascular endothelial growth factor (VEGF)- and fibroblast growth factor-2-induced angiogenesis, and suppressed primary tumor growth and metastasis in the Lewis lung carcinoma mouse model (11). These studies raise the possibility that reduced DHA level may lead to progression of metastasis in patients with RCC, or that increased DHA level may lead to suppression of metastasis.

The effect of DHA on tumor angiogenesis is pivotal, since the hypervascularity, sustained by tumor angiogenesis, is a hallmark of RCC. Current advances in the treatment of metastatic RCC, supported by a better understanding of the RCC biology, are focused on anti-angiogenesis. Although metastasis remains poorly understood, angiogenesis is crucial for the metastatic process. During metastatic dissemination, a cancer cell develops from the proximal tubules and promotes angiogenesis to feed itself and to enter the microvasculature. A cancer cell that survived the immune system translocates through the bloodstream to the microvessels of distant tissues, exits the bloodstream and colonizes in the distant tissues, where angiogenesis is promoted to feed colonized tumor cells and help them proliferate. Hypervascularity is prominent in both primary and metastatic lesions on enhanced CT. RCCs tend to spread either by direct invasion through the renal capsule into the perinephric fat, or by direct extension into the renal vein. Staging of RCC takes into account the presence of renal vein involvement and the prognosis of T3 disease with the presence of renal vein involvement is worse compared with that of T2 disease (23). Angiogenesis is a hallmark of the RCC biology and DHA possesses a variety of anti-angiogenic properties. First, n-3 FAs, including DHA, inhibit the biosynthesis of arachidonic acid-derived eicosanoids, which have pro-inflammatory properties, to promote tumor angiogenesis (4). Eicosanoids produced from n-6 FAs (arachidonic 
acid) stimulate inflammation and tumor angiogenesis, while eicosanoids produced from n-3 FAs, such as EPA and DHA, are anti-inflammatory and do not stimulate angiogenesis. Second, epoxy metabolites of DHA, which are lipid mediators produced by cytochrome P450 epoxygenases, inhibit angiogenesis and metastasis (11). Finally, it was suggested that n-3 FAs exert potent anti-angiogenic effects by inhibiting the production of angiogenic mediators, such as VEGF, platelet-derived growth factor, platelet-derived endothelial growth factor, cyclo-oxygenase 2, prostaglandin E2, nitric oxide, nuclear factor $\kappa \mathrm{B}$, matrix metalloproteinases and $\beta$-catenin (8).

It is also possible that the low DHA level is the result of metastasis or poor cancer-specific survival, resulting in cancer cachexia, which is one of the most common paraneoplastic syndromes associated with RCC. Cachexia is a complex metabolic syndrome associated with underlying illness and characterized by loss of muscle, with or without loss of fat mass (24). Anorexia, inflammation, insulin resistance and increased muscle protein breakdown are the most prominent clinical characteristics of cachexia. DHA is an essential nutrient that cannot be synthesized in the body and must be obtained from the diet. The decreased DHA level in patients with metastasis in this study may be the result of cancer cachexia accompanied by anorexia or lipid metabolism changes, similar to those described in patients with pancreatic cancer (22).

This study has several limitations. First, it was a case-control study including only 112 patients; thus, our results require confirmation by a large randomized control prospective trial. Second, the mechanism underlying the association between low DHA level and metastatic disease or poor cancer-specific survival was not elucidated in this study. We are currently investigating the antitumor effects of DHA, such as antitumor migration and invasion or anti-angiogenesis, to determine whether low DHA level is a causative factor in metastatic disease and shortened cancer-specific survival. However, even with these limitations, our study strongly suggests that DHA may be a useful biomarker to predict prognosis of patients with RCC.

In conclusion, we herein demonstrated that the serum DHA levels in RCC patients with metastasis were lower compared with those in patients without metastasis. In addition, low serum DHA level was an independent predictor of shortened cancer-specific survival in a multivariate Cox proportional hazard model. Our results suggest that the serum DHA level may be a useful biomarker to predict prognosis in patients with RCC and personalize follow-up strategy.

\section{References}

1. Jemal A, Siegel R, Ward E, Hao Y, Xu J, Murray T and Thun MJ: Cancer statistics, 2008. CA Cancer J Clin 58: 71-96, 2008.

2. Bukowski RM: Prognostic factors for survival in metastatic renal cell carcinoma: Update 2008. Cancer 115 (Suppl 10) S2273-S2281, 2009.

3. Sonpavde G and Choueiri TK: Biomarkers: The next therapeutic hurdle in metastatic renal cell carcinoma. Br J Cancer 107: 1009-1016, 2012.

4. Larsson SC, Kumlin M, Ingelman-Sundberg M and Wolk A: Dietary long-chain n-3 fatty acids for the prevention of cancer: A review of potential mechanisms. Am J Clin Nutr 79: 935-945, 2004.

5. Sasazuki S, Inoue M, Iwasaki M, Sawada N, Shimazu T, Yamaji T, Takachi R and Tsugane S; Japan Public Health Center-Based Prospective Study Group: Intake of n-3 and n-6 polyunsaturated fatty acids and development of colorectal cancer by subsite: Japan Public Health Center-based prospective study. Int J Cancer 129: 1718-1729, 2011
6. Terry PD, Rohan TE and Wolk A: Intakes of fish and marine fatty acids and the risks of cancers of the breast and prostate and of other hormone-related cancers: A review of the epidemiologic evidence. Am J Clin Nutr 77: 532-543, 2003.

7. Sonoda T, Nagata Y, Mori M, Miyanaga N, Takashima N, Okumura K, Goto K, Naito S, Fujimoto K, Hirao Y, et al: A case-control study of diet and prostate cancer in Japan: Possible protective effect of traditional Japanese diet. Cancer Sci 95: 238-242, 2004

8. Spencer L, Mann C, Metcalfe M, Webb M, Pollard C, Spencer D, Berry D, Steward W and Dennison A: The effect of omega-3 FAs on tumour angiogenesis and their therapeutic potential. Eur J Cancer 45: 2077-2086, 2009.

9. Serini S, Fasano E, Piccioni E, Monego G, Cittadini AR, Celleno L, Ranelletti FO and Calviello G: DHA induces apoptosis and differentiation in human melanoma cells in vitro: Involvement of HuR-mediated COX-2 mRNA stabilization and beta-catenin nuclear translocation. Carcinogenesis 33: 164-173, 2012.

10. Mandal CC, Ghosh-Choudhury T, Yoneda T, Choudhury GG and Ghosh-Choudhury N: Fish oil prevents breast cancer cell metastasis to bone. Biochem Biophys Res Commun 402: 602-607, 2010.

11. Zhang G, Panigrahy D, Mahakian LM, Yang J, Liu JY, Stephen Lee KS, Wettersten HI, Ulu A, Hu X, Tam S, et al: Epoxy metabolites of docosahexaenoic acid (DHA) inhibit angiogenesis, tumor growth, and metastasis. Proc Natl Acad Sci USA 110: 6530-6535, 2013.

12. Rogers KR, Kikawa KD, Mouradian M, Hernandez K, McKinnon KM, Ahwah SM and Pardini RS: Docosahexaenoic acid alters epidermal growth factor receptor-related signaling by disrupting its lipid raft association. Carcinogenesis 31: $1523-1530,2010$

13. Patterson RE, Flatt SW, Newman VA, Natarajan L, Rock CL, Thomson CA, Caan BJ, Parker BA and Pierce JP: Marine fatty acid intake is associated with breast cancer prognosis. J Nutr 141: 201-206, 2011.

14. Vaughan VC, Hassing MR and Lewandowski PA: Marine polyunsaturated fatty acids and cancer therapy. Br J Cancer 108: 486-492, 2013

15. Bougnoux P, Hajjaji N, Ferrasson MN, Giraudeau B, Couet C and Le Floch O: Improving outcome of chemotherapy of metastatic breast cancer by docosahexaenoic acid: A phase II trial. Br J Cancer 101: 1978-1985, 2009.

16. Lopez-Beltran A, Scarpelli M, Montironi R and Kirkali Z: 2004 WHO classification of the renal tumors of the adults. Eur Urol 49: 798-805, 2006

17. Sattler W, Puhl H, Hayn M, Kostner GM and Esterbauer H: Determination of fatty acids in the main lipoprotein classes by capillary gas chromatography: BF3/methanol transesterification of lyophilized samples instead of Folch extraction gives higher yields. Anal Biochem 198: 184-190, 1991.

18. Gleissman H, Segerström L, Hamberg M, Ponthan F, Lindskog M, Johnsen JI and Kogner P: Omega-3 fatty acid supplementation delays the progression of neuroblastoma in vivo. Int J Cancer 128: 1703-1711, 2011.

19. Surette ME: The science behind dietary omega-3 fatty acids. CMAJ 178: 177-180, 2008.

20. Zuijdgeest-van Leeuwen SD, van der Heijden MS, Rietveld T, van den Berg JW, Tilanus HW, Burgers JA, Wilson JH and Dagnelie PC: Fatty acid composition of plasma lipids in patients with pancreatic, lung and oesophageal cancer in comparison with healthy subjects. Clin Nutr 21: 225-230, 2002.

21. Cvetković Z, Vucić V, Cvetković B, Petrović M, Ristić-Medić D, Tepsić J and Glibetić M: Abnormal fatty acid distribution of the serum phospholipids of patients with non-Hodgkin lymphoma. Ann Hematol 89: 775-782, 2010.

22. Macášek J, Vecka M, Žák A, Urbánek M, Krechler T, Petruželka L, Staňková B and Zeman M: Plasma fatty acid composition in patients with pancreatic cancer: Correlations to clinical parameters. Nutr Cancer 64: 946-955, 2012.

23. Kim SP, Alt AL, Weight CJ, Costello BA, Cheville JC, Lohse C, Allmer C and Leibovich BC: Independent validation of the 2010 American Joint Committee on Cancer TNM classification for renal cell carcinoma: Results from a large, single institution cohort. J Urol 185: 2035-2039, 2011.

24. Ebadi M and Mazurak VC: Evidence and mechanisms of fat depletion in cancer. Nutrients 6: 5280-5297, 2014. 